\title{
HISTORIA DEL CÓDIGO DE COMERCIO PERUANO
}

\author{
Miguel TorRes MéndeZ
}

«Si los hombres de genio son cordilleras nevadas, los imitadores no pasan de riachuelos alimentados con el deshielo de la cumbre.»

Manuel González Prada

\section{Introducción}

Cuando se tiene que investigar sobre la historia de esta área del Derecho peruano, el área del Derecho comercial referida a la codificación que la regula, nada resulta más incoherente y decepcionante. Estos dos calificativos, tan contundentes y lapidarios, serán ampliamente demostrados y justificados en este trabajo.

Para esto se debe señalar que el desarrollo de este trabajo va a estar dividido en dos partes. Una primera "parte expositiva», en la que se hará una mera exposición de los hechos, tal como acaecieron históricamente, y una segunda "parte denunciativa», en la que se emitirán juicios valorativos y análisis críticos sobre estos hechos que enmarcan la historia de la codificación mercantil peruana.

\section{Parte expositiva}

Para investigar cuál ha sido la historia del vigente Código de Comercio peruano hay que remontarse al siglo pasado. $Y$ no porque este código tenga como fecha de inicio de vigencia el año de 1902 y esto lleve a pensar entonces que, por tanto, fue durante los años próximos anteriores a 1902 durante los cuales se preparó o se realizó la elaboración o redacción de este 
código de comercio, sino porque, en realidad, como se comprobará más adelante, dicho código es del siglo pasado, aun cuando tenga como fecha de inicio de vigencia el año de 1902.

Allá por el año de 1895, exactamente el 6 de noviembre de ese año, fue presentado a la entonces Cámara de Diputados un proyecto de ley por los diputados Felipe de Osma, José M. Manzanilla, Germán Leguía, M. J. Pozo, J. D. Cáceres y Washington Ugarte, que proponía la adopción del Código de Comercio español de 1885 y la elección de una comisión que informara acerca de las reformas que fuese necesario introducir en él.

Se puede apreciar bien, entonces, que después de diez años de la puesta en vigencia del mencionado Código de Comercio español de 1885 , ya en el Perú se proponía la incorporación de este código en su normatividad.

Pero esta observación no debe causar sorpresa porque en ese año, 1895, se encontraba vigente el Código de Comercio de 1853 que era resultado a su vez de la incorporación del Código de Comercio español de 1829 a la normatividad peruana. De tal manera que cuando en 1895 se proponía la incorporación de un código extranjero a la normatividad peruana, ya en el Perú existía un precedente.

Tal parece que en 1895 los «legisladores» peruanos al ver que ya en España habían cambiado de código de comercio derogando el anterior de 1829, se hacía necesario también aquí entonces, por lógica, tener que hacer los mismo; es decir, cambiar de código de comercio. Pues hubiese sido ilógico e inconsecuente continuar utilizando el Código de Comercio de 1853, que era el mismo de España de 1829 y que ya había sido derogado y sustituido por otro en dicho país diez años atrás en 1885 .

Estas circunstancias han debido ser sin duda las que motivaron a los diputados anteriormente mencionados a proponer en 1895 la adopción de un nuevo código de comercio en el Perú.

Lo que sucedió a continuación entonces fue que la propuesta de estos diputados fue tomada en cuenta, pues de acuerdo a los datos obtenidos de los propios dictámenes legislativos con los que se discutió y aprobó la adopción del Código de Comercio de 1902, después de la presentación del pro- 
yecto de ley —en el que se proponía la adopción del Código de Comercio español de 1885 - en 1895, hubo de transcurrir dos legislaturas para que finalmente se presentara el dictamen con el que se aprobó la incorporación de dicho código a la normatividad peruana. Fue en las sesiones del año 1897 en las que ese dictamen fue ampliamente discutido y aprobado, quedando así, por tanto, aceptada la propuesta de adopción del Código de Comercio español de $1885^{\perp}$.

Pero este proyecto de ley no llegó a ser ley del Estado porque en ese año (1897) se clausuró el Congreso sin que el Senado hubiera tenido tiempo para ocuparse del asunto. Fue entonces cuando a comienzos del año siguiente (1898), como el Congreso estaba inactivo, tomó riendas en el asunto el Poder Ejecutivo, pues éste participaba en la misma medida de las aspiraciones de los diputados que habían presentado el proyecto de ley. El Poder Ejecutivo (exactamente el gobierno de Nicolás de Piérola) expidió entonces un decreto el 28 de febrero de 1898 mediante el cual se nombró a los doctores Luis Felipe Villarán y Felipe de Osma y Pardo miembros de la comisión encargada de adaptar el Código de Comercio español de 1885 a nuestras costumbres mercantiles, forma de gobierno y organización de los juzgados y tribunales, disponiendo a su vez que la Cámara de Comercio de Lima agregara un comerciante, quien unido a los dos anteriores completaría dicha comisión.

De esta manera quedó conformada completamente esta comisión, a la que precisamente dicho decreto denominó «Comisión encargada de adaptar el Código de Comercio español de 1885 a nuestras costumbres mercantiles, forma de gobierno y organización de los juzgados y tribunales»², comisión que fue creada por el decreto del 28 de febrero de 1898 y que fue propuesta por el proyecto de ley que fue presentado por los diputados ya mencionados, en 1895.

Según datos también de los dictámenes legislativos, esta comisión «trabajó asiduamente durante ocho meses» después de los cuales presentó el

1 Lama, Miguel de la,«Dictámenes legislativos del Congreso», en Código de Comercio, Lima, 1902, pág. 219.

2 Lama, Miguel de la, op. cit., pág. 220. 
Código al Poder Ejecutivo y éste lo sometió a la Cámara de Diputados del mismo año (1898) ${ }^{3}$. Pero no hubo tiempo en esa legislatura ni en la ordinaria de ese año para tratar esa materia, razón por la cual iba a demorar más el proceso de aprobación del Código. Fue entonces cuando intervino la Cámara de Comercio de Lima, que estaba nuy interesada en la pronta aprobación, y presentó en 1899 al Poder Ejecutivo una exposición en la que pedía se sometiera el Código al Congreso extraordinario que estaba funcionando en aquel momento.

El Poder Ejecutivo accedió y remitió el Código al Congreso extraordinario, para lo cual se formó en éste una Comisión auxiliar de legislación que se encargaría de examinar el Código. Este hecho podría llevar a pensar entonces que la «adaptación» que hizo la comisión encargada para ello fue muy «técnica» y «elaborada», pues para su revisión el Congreso se vio en la necesidad de crear una Comisión auxiliar de legislación. En la segunda parte de este trabajo se comprobará si ello fue realmente así.

La Comisióm auxiliar de legislación se dedicó entonces a examinar el Código. Y la revisión que hizo dio como resultado tres dictámenes legislativos que son precisamente de los cuales se está obteniendo toda esta información. El primero de estos dictámenes fue un dictamen de mayoría y el segundo uno de minoría. Ambos fueron emitidos con fecha 1 de diciembre de 1899. Y el tercer y último dictamen fue un dictamen concordado emitido con fecha 5 de diciembre de ese mismo año.

En el primer dictamen, el de mayoría, la Comisión auxiliar de legislación simplemente reprodujo las consideraciones que expusieron los miembros de la Comisión encargada de adaptar el Código de Comercio español para emprender su obra, las mismas que teminaban con la propuesta de varias conclusiones. De estas conclusiones las más importantes eran la aprobación del proyecto de Código de Comercio preparado por esta última comisión, la autorización al Poder Ejecutivo para la publicación del Código y que la fecha de inicio de vigencia fuera el 17 de marzo de 1900.

Pero en el segundo dictamen, el de minoría, la Comisión auxiliar de legislación expuso que había hecho un estudio exhaustivo de la necesidad

3 Ibidem, pág. 220. 
de la reforma del Código de Comercio en el Perú, de cómo y por qué se había dirigido esta reforma a la adopción del Código de Comercio español de 1885, así como expuso las modificaciones que hizo a este código la Comisión encargada de adaptarlo y las principales reformas que finalmente este nuevo código introduciría en la legislación vigente de ese entonces.

El segundo dictamen finalizaba también con la propuesta de varias conclusiones. De ellas las más importantes eran la aprobación del proyecto de Código de Comercio presentado por el Poder Ejecutivo y que la fecha de inicio de vigencia fuese el 18 de marzo de 1900 porque ése era el día de la apertura de los tribunales en el siguiente año judicial de 1900 .

Es de mucha importancia destacar que en estos dos dictámenes legislativos la Comisión auxiliar de legislación del Congreso empezó a emitirlos haciendo notar, y por ende criticando, la injustificada, para ellos, falta de Exposición de motivos en este proyecto de Código de Comercio. Así, en el dictamen en minoría se observó lo siguiente: «esta clase de obras no deben venir como ahora, sin una exposición de motivos que facilite su estudio y comprensión»". De la misma forma, en el dictamen en mayoría también se observó lo siguiente: «Habiéndose omitido por ellos, la exposición de motivos que debía anteceder al trabajo de aliento que emprendieron ${ }^{5}$. En la segunda parte de este trabajo se comprobará también si era injustificada o no la falta de exposición de motivos, así como si fue realmente un «trabajo de aliento».

A través del dictamen concordado la mencionada comisión presentó igualmente varias conclusiones, siendo la primera y más trascendente de ellas la de aprobación del proyecto de Código de Comercio que había remitido el Poder Ejecutivo. De tal manera que lo único que en realidad hizo esta comisión a través de dicho dictamen fue simplemente, pues, aprobar el proyecto de Código de Comercio.

Como siguiente paso dicho proyecto fue remitido a la Cámara de Senadores de ese entonces para su correspondiente revisión y aprobación, para

4 Lama, Miguel de la, op. cit., pág. 217.

5 Ibidem, pág. 185. 
lo cual emitió también su respectivo dictamen. Pero dicha revisión, aprobacion y dictamen senatorial no se realizaron inmediatamente, sino luegb de nueve meses. Así es como, recién con fecha 1 de setiembre de 1900 , sèexpide el dictamen del Senado.

En el dictamen del Senado éste también propone, al igual que en los demás dictámenes, varias conclusiones; siendo la primera y más trascendente de ellas la de aprobación de las conclusiones venidas en revisión de la Cámara de Diputados con excepción de sólo algunas normas que se indicaron.

Nuevamente es muy significativo y destacable el hecho de que también en este dictamen se observó la falencia de exposición de motivos de este proyecto de codificación mercantil: "Tarea muy fácil sería juzgar su trabajo, si estuviera acompañado de una motivación más o menos extensa, o siquiera de una expocisión de plan, que ayudase a su estudio» ${ }^{6}$.

Pero aun con esta última aprobación efectuada por el Senado en 1900 no pudo llegar todavía a entrar en vigencia este código de comercio. Ello se debió a que el Congreso consideró conveniente discutirlo y revisarlo todavía en las sesiones parlamentarias de las siguientes legislaturas del año 1901. Durante este año las conclusiones de los tres dictámenes fueron, pues, discutidas, revisadas y finalmente aprobadas en sesiones parlamentarias tanto de la Cámara de Diputados como de la Cámara de Senadores de ese entonces.

Finalmente, transcurridas las legislaturas del año 1901 el Congreso remitió el proyecto de Código de Comercio al Poder Ejecutivo para su promulgación. Es así como finalmente el Poder Ejecutivo de ese entonces, exactamente el gobierno de Eduardo López de Romaña, promulgó el Código de Comercio el 15 de febrero de 1902 disponiéndose que su vigencia comenzara a regir el 1 de julio de ese mismo año, como efectivamente rige hasta nuestros días. Este lapso de meses entre la promulgación y la puesta en vigencia obedeció a que se consideró que se requería algún tiempo para su necesaria difusión y divulgación, tal como siempre se estila hasta la fecha con las leyes de importancia como son los Códigos.

6 Lama, Miguel de la, op. cit. pág. 231. 


\section{Parte denunciativa}

En la introducción se señaló, adelantando juicios valorativos y críticas, que la historia de la codificación mercantil peruana resulta ser «incoherente» y «decepcionante». Pues bien, ha llegado el momento de demostrar y jusitificar estos calificativos.

Como bien se puede apreciar, la historia de la codificación mercantil peruana está caracterizada por incorporaciones de códigos extranjeros; 0 , como lo llamaron los que incorporaron estos códigos, por «adaptaciones» de códigos extranjeros a la normatividad peruana. Desde que el Perú es una república han regido en este país dos códigos de comercio. El primero, como ya se ha mencionado, fue el Código de Comercio de 1853 que fue resultado de la incorporación del Código de Comercio español de 1829 a la normatividad peruana. Y el segundo es el que rige actualmente desde 1902 y que es el resultado a su vez de la incorporación del Código de Comercio español de 1885 , como ya se ha expuesto y estudiado en la primera parte de este trabajo.

De acuerdo a esta situación, es fácil observar que en el Perú nunca se ha elaborado o «creado» un código de comercio. Lo único que aquí se ha hecho es incorporar o «adaptar», de acuerdo a la terminología utilizada por los que participaron en esta incorporación, los códigos de comercio de otro país. El Perú, en consecuencia, no ha tenido códigos de comercio propios o autóctonos. Los códigos de comercio que ha tenido han sido siempre códigos extranjeros, han sido siempre los códigos de comercio de España.

Ahora bien, para poder fundamentar y justificar bien todos los juicios y críticas que puedan vertirse sobre esta situación, es importante indagar y determinar cuál ha sido o cuáles han sido las razones por las que en el Perú se procedió de esta manera en la codificación mercantil. Es decir, se deben averiguar las razones por las que en el Perú se prefirió siempre copiar códigos de comercio en lugar de crear o elaborar uno propio. Pues fácil, cómodo e ilogico sería formular críticas sin haber previamente analizado cuál fue el trasfondo del asunto. De esta manera una vez determinadas las razones que condujeron a los «legisladores» mercantiles peruanos a proceder de esta forma, se analizará si son o no justificadas las mismas. 
Se debe determinar entonces, en primer lugar, las razones que la Comisión encargada de adaptar el Código de Comercio español de 1885 expuso para decidir realizar la adaptación de este código de comercio extranjero. Estas razones fueron dos y se pueden encontrar en las consideraciones que esta comisión expuso para emprender la obra encomendada y que la Cámara de Diputados de ese entonces reprodujo en su primer dictamen legislativo.

La primera razón que esta comisión expuso fue la siguiente: «El Código de Comercio español de 1885 , justo medio, transacción entre las doctrinas que informaron el de 1829 del mismo país, y las novísimas influenciadas por el espíritu del Derecho anglo-americano, conviene al Perú: entre unas y otras hay considerable distancia, la que no es posible que la recorran en toda su extensión países jóvenes como el Perú, impremeditadamente, sino por grados, por etapas. Mejora considerable conseguirá el Perú, con la adopción del mencionado Código de 1885 , que se distingue por las tendencias que se han enunciado»?

Y la segunda razón, a su vez, fue la siguiente: «Aunque Maine ha dicho con profundo sentido que el derecho internacional por excelencia es el mercantil; si es verdad que éste contiene preceptos que, a diferencia de las leyes civiles, se desinfluencian de circunstancias de clima, geografía, etc.; si ellos armonizan casi hasta tocar en la unidad; no se puede que negar que los mismos, concretados en artículos de los Códigos se diferencian por razones de sistema: el nuestro, de calificación subjetiva, no puede sin graves tropiezos, variarse absolutamente por la objetiva. Ésta es otra razón, que en concepto de los infrascritos, justifica la adopción del Código de Comercio español de $1885 »^{8}$.

Como se puede apreciar, ninguna de estas dos razones tomó en consideración si este código de comercio extranjero era acorde o ajustado a la realidad comercial peruana de aquella época. En otras palabras, al adoptarse este código de comercio extranjero no se tomó en cuenta o estudió la realidad comercial peruana.

\footnotetext{
7 Lama, Miguel de la, op. cit., pág. 188.

8 Ibidem, pág. 188.
} 
Pues bien, se tiene en primer lugar que, de acuerdo a la primera razón, por el hecho de que el Código de Comercio español de 1885 era -en consideración de los miembros de esta comisión- «el punto intermedio» entre viejas doctrinas (que informaron el Código de Comercio español de 1829) y nuevas doctrinas (que el Derecho anglo-americano influencia), era conveniente copiar este código.

¿Pudo ser ésta en realidad una razón válida para incorporar otro código de comercio extranjero y no elaborar uno propio? ¿Era preferible acaso copiar un código ya hecho que elaborar uno nuevo por esta razón, de ser el ya hecho un «punto intermedio» entre dos corrientes doctrinarias?

Igualmente, ¿era una buena razón para copiar el Código de Comercio español de 1885 el que el código anterior (que era el mismo de España de 1829) era de calificación subjetiva, y, por lo tanto, el variarlo absolutamente a través de una calificación objetiva iba a traer graves tropiezos?

Estas razones, en realidad, no justifican ni demuestran en modo alguno que en el Perú existió la necesidad de incorporar o copiar este código de comercio extranjero, por más que éste signifique o sea un punto intermedio entre dos corrientes doctrinarias. Lo apropiado, coherente y razonable hubiese sido que se hubiera elaborado un código de comercio propio en el que se tomaran en cuenta esas viejas y nuevas corrientes doctrinarias, pero en el que fundamentalmente se tomara en cuenta la realidad comercial peruana.

Resulta evidente darse cuenta entonces que los «legisladores» peruanos prefirieron hacer lo más fácil. Pues, evidentemente, lo más fácil (y ocioso también) era hacer lo que finalmente se hizo: copiar un código de comercio extranjero ya hecho en vez de hacer uno propio. Porque elaborar un código de comercio propio significaba (y significa aún) estudiar la realidad comercial peruana, las nuevas instituciones mercantiles que se han ido creando y cómo han ido evolucionando las que ya existen. Todo ello analizando también las nuevas y viejas corrientes doctrinarias que puedan ser útiles de tomarse en cuenta en un cuerpo legal de esta naturaleza.

$Y$ todo esto, por supuesto, implicaba emprender una concienzuda y esforzada labor de estudio y creación que, tal parece, los «legisladores» peruanos no se sintieron en capacidad de llevar a cabo. Al no hacer esto últi- 
mo dichos «legisladores», evidentemente se prefirió o prefirieron injustificadamente lo más fácil: copiar simplemente un código de comercio extranjero.

Habiéndose comprobado que las razones expuestas por los miembros de la Comisión encargada de adaptar el Código de Comercio español de 1885 no justificaban ni, por ende, demostraban que en el Perú hubo la necesidad de copiar este código, se pasará ahora a analizar si las razones que a su vez expusieron los parlamentarios para incorporar dicho código justificaban realmente esta incorporación.

La Cámara de Diputados de aquel entonces expuso la siguiente razón: «El año 1829 arreglo España su Código de Comercio, y el año 1853 nosotros lo aceptamos con ligeras modificaciones, atendiendo a la unidad de costumbres, de idioma y de tendencias. España encontró el año 1885 deficiente su ley comercial, así como hoy juzgamos la nuestra, y emprendió la tarea de la reforma, coronando su obra con un nuevo Código, que aunque no adopta de lleno las ideas modernas, responde, sin duda, a las necesidades mercantiles de la época. Con estos antecedentes, y en la necesidad de emprender nosotros la reforma, natural y lógico es partir de aquella base ya estudiada y aceptada en circunstancias tan análogas a las nuestras»".

Por su parte, la Cámara de Senadores, también de aquel entonces, expuso la siguiente razón: «Desde luego, la Comisión opina, que de no hacerse un Código propio para el Perú, lo mejor ha sido, sin duda, adoptar el español. De esta suerte conservaremos nuestra ley comercial, el espíritu de tradicion, factor muy apreciable de buena codificación» ${ }^{10}$.

Como se puede advertir, estas dos razones consisten básicamente en considerar que el Código de Comercio español de 1885 sí era apropiado para el Perú por las situaciones de tradición, igualdad de costumbres, idioma, tendencias, y porque España había adoptado este código en circunstancias análogas a las que el Perú atravesaba en ese entonces.

De todas estas situaciones que conforman estas dos razones, resulta sumamente claro y evidente que la única valedera y con asidero fue la de la

9 LAMA, Miguel de la, op. cit., pág. 187.

10 Ibidem, pág. 187. 
tradición. Porque en realidad sí era verdaderamente tradición en el Perú copiar o incorporar códigos extranjeros, al menos en cuanto a codificación mercantil se refiere. No otra cosa fue el código de comercio anterior, que rigió en el Perú desde 1853 hasta 1902, que una copia fiel del Código de Comercio español de 1829.

Como esto era «tradición», los diputados de aquel entonces invocándola justificaban la incorporación del nuevo Código de Comercio español de 1885 a la normatividad peruana. Habrá que preguntarnos ahora, nosotros los peruanos actuales, si debemos enorgullecernos de esta tradición o si, por el contrario, debemos avergonzarnos de ella.

Pero de ninguna manera resultan ser válidas las demás situaciones que conforman estas dos razones expuestas por el Parlamento de aquel entonces. El hecho de que España había promulgado su nuevo Código de Comercio de 1885 en circunstancias análogas a las que el Perú atravesaba a finales del siglo pasado, no fue sino más que una lógica e inevitable consccucncia. Ello se debe a que España promulgó un nuevo código de comercio porque su anterior Código de 1829 había devenido obsoleto, y, por lo tanto, inútil. Y como el anterior Código de Comercio «peruano» de 1853 era el mismo de España de 1829 (pues fue una copia exacta de este último código); resultaba evidente entonces que dicho primer Código de Comercio "peruano» había devenido también obsoleto y se hacía necesario también, en consecuencia, tener que cambiarlo.

En otras palabras, lo que ocurrió en España (la adopción de un nuevo código de comercio) tenía necesaria e inevitablemente que ocurrir también aquí en el Perú; pues el código de comercio que aquel país derogó por obsoleto era exactamente el mismo que regía en el Perú por ser el de este último copia textual de aquél. Por tal razón, esas «circunstancias análogas» que atravesaron tanto España como el Perú al adoptar la primera un nuevo código de comercio sólo pueden justificar que en el Perú también se necesitaba cambiar de código de comercio, pero no pueden justificar que hubo necesidad de copiar otra vez otro código de comercio español.

Por último, como obvio resulta darse cuenta, tampoco justifica la copia de este código de comercio extranjero el hecho de que el Perú haya tenido las mismas costumbres, tendencias y el mismo idioma. Estas razones, en 
realidad, resultan ser sumamente absurdas. El que un país hable el mismo idioma que el de otro país no puede ser de ninguna manera una razón para que el primero copie o adopte la legislación comercial del segundo. Seguir un razonamiento como éste es realmente absurdo porque ello implicaría entonces que también el Perú debe adoptar el Código Civil, Penal, Tributario, etc., de España, lo cual, pues, no tiene sentido.

$\mathrm{Y}$ con respecto a las mismas costumbres y tendencias, éstas pudieron haber sido semejantes en aquella época, pero no de manera absoluta como para justificar la copia «textual», «absoluta» del Código de Comercio español de 1885. Así como no puede haber dos seres exactamente iguales, tampoco puede haber dos realidades comerciales exactamente iguales. Por tanto, por el hecho de que el Perú en aquella época haya tenido ciertas costumbres y tendencias comunes con España, éstas tampoco justificaban realmente realizar una copia «textual» del Código de Comercio de aquel país.

El hecho de que lo que en realidad se hizo legislativamente aquí en el Perú fue nada más que copiar un código extranjero, fue la razón, pues, por la que el Código de Comercio «peruano» que fue resultado de dicha copia no tuviera exposición de motivos. ¿Qué exposición de motivos puede tener este código si no es en realidad creación de una ley propia (respecto de la cual los autores o verdaderos legisladores pueden expresar los fines o motivos que tuvieron para crearla) sino, por el contrario, es sólo la copia de una ley ya creada?

Por eso resulta sumamente «irónico», por no decir más, el comentario o apreciación que tuvieron a bien hacer en su momento los parlamentarios de ese entonces al revisar este código de comercio cuando era sólo un proyecto. Tal apreciación fue la siguiente: «La Comisión, en concepto de los infrascritos, ha redactado un Código de Comercio privado nacional» ${ }^{11}$. Como resulta evidente objetar: ¿de qué «redacción» se habla si lo que se hizo fue una mera copia o calco? Igualmente, ¿cómo puede ser Código de Comercio «nacional» si en realidad se trata de uno extranjero, se trata del Código de Comercio español de 1885 ?

11 Lama, Miguel de la, op. cit., pág. 188. 
De tal manera que puede advertirse ahora que la falta de exposición de motivos de dicho código, que tanto fue criticada por los parlamentarios de aquel entonces, sí era justificada. No podía ser de otra manera, al no haber sido creación propia, sino sólo copia.

Ahora bien, los reclamos que de dicha exposición de motivos hicieron dichos parlamentarios demuestran dos situaciones muy negativas y reprobables que se deben resaltar en este inusitado proceso legislativo que se dio en el Perú en cuanto a la codificación mercantil.

La primera de ellas lo constituye la ignorancia por parte de estos parlamentarios del texto del Código de Comercio español de 1885, pues no advirtieron que el código que se proponía adoptar aquí no contenía modificación ni adaptación alguna de aquél. Al reclamar una exposición de motivos creen que sí hay modificaciones y, por ende, verdadera adaptación que sea necesaria motivar o justificar. De manera que esto también demuestra desidia y negligencia por parte de ellos al no haberse dado el mínimo trabajo de cotejar o comparar los textos de dichos códigos, lo cual no implicaba ninguna dificultad. ¿Qué clase de trabajo de revisión hicieron entonces si ni siquiera pudieron darse cuenta de esta situación?

Pero lo que es más grave aún es la actitud que asumieron los miembros de la primera comisión frente a esta negligencia de los parlamentarios, lo cual constituye la segunda situación negativa que siguió a ésta que se acaba de mencionar. Frente a los reclamos de exposición de motivos por parte de los parlamentarios, que demostraban la falsa apreciación de éstos de que esa comisión había efectuado una verdadera labor de modificación y adaptación, los miembros de dicha comisión no se dignaron o tuvieron la entereza de aclarar que en realidad su trabajo no consistió en esa labor, sino simplemente en una simple copia. Hubo, pues, incluso una actitud de mala fe por parte de estos «legisladores» peruanos. Al parecer, la razón de esta reprobable actitud consistió en que no les convenía pasar a la historia como simples copiadores, sino como verdaderos legisladores.

Asimismo, puede advertirse también que por haberse tratado la adopción de este código de una simple copia, fue absolutamente innecesario entonces haber dedicado tanto tiempo para esa labor. Igualmente, también fue innecesaria la creación en el Congreso de una «Comisión auxiliar de legislación» encargada de la revisión del trabajo de adaptación que supuestamente 
iba a realizar la primera comisión que se creó para tal fin (llamada pomposamente «Comisión encargada de adaptar el Código de Comercio español de 1885 a nuestras costumbres mercantiles, formas de gobierno y organización de los juzgados y tribunales»).

Así pues, ¿era justificado acaso dedicar siete años (1895-1902) para tan sólo copiar una ley ya hecha? Del mismo modo, como no hubo en realidad una labor de «adaptación» del Código de Comercio español de 1885 , sino solamente una burda copia del mismo, ya no era necesario entonces que se constituyera otra comisión especial para que se dedicara a revisar el trabajo realizado por la primera. ¿Se justifica acaso realizar una labor de revisión de una simple copia o calco?

Esto demuestra también que la labor realizada por dicha primera comisión no fue en absoluto técnica ni muy elaborada. Pues, ¿puede ser acaso una labor de copia o calco técnica y elaborada? De tal manera que aquella apreciación según la cual dicha comisión «trabajó asiduamente durante ocho meses» no es más que una ingenua e irónica apreciación que no corresponde en absoluto a la realidad.

Pues bien, con todo lo expuesto y demostrado en la segunda parte de este trabajo quedan ahora sí plenamente justificados los calificativos que se atribuyeron en la introducción del mismo a la historia de nuestro cuerpo legal comercial. La historia de cómo surgió y se plasmó el Código de Comercio de 1902 resulta ser en realidad «incoherente» y «decepcionante». ¿Cómo no va a ser incoherente el que la historia de la legislación mercantil peruana esté constituida sólo por copias de códigos de comercio extranjeros? Y, ¿cómo no va a ser decepcionante la manera en que actuaron los «legisladores» peruanos?, la cual tiene que ver más con ociosidad, negligencia y mala fe.

Ahora bien, es cierto que la copia del Código de Comercio español de 1885 no fue total, sino que algunas partes de este código de comercio no llegaron a copiarse, como señala Carlos Torres y Torres Lara: «El nuevo Código de 1902 fue a su vez copia del Código español de 1885, aunque con algunos cambios importantes». ${ }^{12}$

12 Torres y Torres Lara, Carlos, «La codificación comercial en el Perú. De un 
Esta situación podría llevar a pensar entonces que en las partes que no se copiaron los «legisladores» peruanos sí crearon o elaboraron normas propias. Sin embargo, la realidad es la misma: otra burda copia. Estas partes si bien no fueron tomadas del Código de Comercio español, fueron copiadas o tomadas sencillamente de otros códigos de comercio extranjeros. Así, la parte sobre letras de cambio fue copiada del Código de Comercio italiano y las partes sobre rematadores y martilleros y sobre cuenta corriente fueron tomadas del Código de Comercio argentino.

Por todo lo expuesto y demostrado, considero que debe hacerse una salvedad o excepción a la conclusión que a llega Fernando de Trazegnies Granda sobre el Derecho peruano del siglo pasado: «El Derecho peruano del siglo XIX parece el resultado de la actividad de un bricoleur intelectual, para usar un término de Levi Strauss (aunque con un sentido algo diferente) $\gg^{13}$. Tal salvedad o excepción debe ser en cuanto al Derecho comercial. En realidad, es demasiado generoso el calificativo de el «resultado de la actividad de un bricoleur intelectual» para esta rama del Derecho peruano, porque realmente ni siquiera se utilizaron elementos sobrantes - como se hace en la actividad de un «bricoleur intelectual»-, sino que se incorporó todo un material no para «construir» (porque no se creó nada) un nuevo objeto de uso práctico, sino para «calcar» simplemente un viejo objeto de uso práctico.

Cabe entonces finalizar el presente trabajo concluyendo que en realidad no existe codificación mercantil «peruana»; dicha codificación es sólo incorporaciones de códigos de comercio extranjeros. La que se suele y se hace llamar como «Codificación mercantil peruana» no es más que una burda copia de codificaciones mercantiles extranjeras.

Por tal razón, a título de reflexión concluyo este trabajo reproduciendo las palabras que un gran estudioso del Derecho mercantil, Joaquín Garrigues, dijera alguna vez: «No es el Derecho de los juristas de gabinete el que a mí me atrae; ese Derecho paralizado en las leyes y en los escrito-

código formal a un código real», en Libro homenaje a Ulises Montoya Manfredi, Lima, 1989, pág. 366.

13 Trazegnies Granda, Fernando de, La idea de Derecho en el Perú republicano del siglo XIX, Lima, 1979, pág. 366. 
rios de los juristas, ese Derecho desarraigado de la tierra sobre la que los hombres se mueven con sus miserias y sus grandezas ${ }^{14}$. Cuán pertinentes y exactas resultan estas últimas palabras de este gran jurista para el caso de la codificación mercantil peruana. El Derecho mercantil peruano es en realidad un Derecho desarraigado de la tierra sobre la que los peruanos se mueven.

14 GaRrigues, Joaquín, «Reto sin respuesta», en Libro homenaje a Javier Zubiri, Madrid, 1970. 